INPLASY

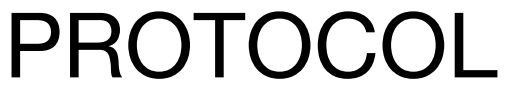

To cite: Gu et al. Chinese herbal medicines for mild cognitive impairment: $A$ protocol for meta-analysis and trial sequential analysis. Inplasy protocol 202190006. doi:

10.37766/inplasy2021.9.0006

Received: 02 September 2021

Published: 02 September 2021

Corresponding author:

Canxing Yuan

cxyuan1964@163.com

Author Affiliation:

Longhua Hospital, Shanghai University of Traditional

Chinese Medicine.

Support: National Key R \& D Program.

Review Stage at time of this submission: Preliminary searches.

Conflicts of interest: None declared.

\section{Chinese herbal medicines for mild cognitive impairment: A protocol for meta-analysis and trial sequential analysis}

Gu, SC1; Zhang, LM²; Wang, CX3; Qu, YJ4; Ma, JY5; Zhen, RR6; Gu, C7; Yuan, CX8.

Review question / Objective: The primary objective of this meta-analysis is to quantify the overall effect of CHMs on global cognitive function in patients diagnosed with $\mathrm{MCl}$. Secondary objectives are to (1) determine whether CHMs positively influence $A D L$ of $\mathrm{MCl}$ patients, (2) evaluate the efficacy of CHMs on mood, and (3) assess the safety and tolerability of the CHMs based on reported adverse events and dropouts.

Main outcome(s): The change in global cognitive function scores will be applied as the primary outcome measure, derived from MMSE, MoCA, and ADAS-cog scales. Secondary outcomes include assessments of $A D L$ and mood which might be measured by the Bayer $A D L$, Erlangen $A D L$, Alzheimer Disease Cooperative Study (ADCS) ADL, the Disability Assessment for dementia (DAD) ADL scales, and Geriatric Depression Scale (GDS). The incidences of adverse events related to CHMs will also be included as secondary outcomes. All outcome measures have to be administered at baseline and directly after the intervention period.

INPLASY registration number: This protocol was registered with the International Platform of Registered Systematic Review and Meta-Analysis Protocols (INPLASY) on 02 September 2021 and was last updated on 02 September 2021 (registration number INPLASY202190006).

\section{INTRODUCTION}

Review question / Objective: The primary objective of this meta-analysis is to quantify the overall effect of CHMs on global cognitive function in patients diagnosed with $\mathrm{MCl}$. Secondary objectives are to (1) determine whether CHMs positively influence ADL of $\mathrm{MCl}$ patients, (2) evaluate the efficacy of CHMs on mood, and (3) assess the safety and tolerability of the CHMs based on reported adverse events and dropouts. Participants diagnosed with $\mathrm{MCl}$ by any proper clinical criteria not caused by traumatic brain injury or space-occupying lesion will be 
included. Interventions in the treatment group will include any kinds of orally administered CHMs such as prescription and Chinese patent medicines in the form of liquids, pills, powders, granules, or capsules as mono-therapy. The control group was defined as patients who were assigned to receive placebo. CHMs in combination with other conservative treatments such as cognitive rehabilitation and oral drugs, and other Traditional Chinese medicine (TCM) treatments such as intravenous medication, acupuncture, and massage for cognitive deficit will be excluded. The concomitant use of medication for noncognitive disorders will be allowed. The change in global cognitive function scores will be applied as the primary outcome measure, derived from MMSE, MoCA, and ADAS-cog scales. Secondary outcomes include assessments of ADL and mood which might be measured by the Bayer ADL, Erlangen ADL, Alzheimer Disease Cooperative Study (ADCS) ADL, the Disability Assessment for dementia (DAD) ADL scales, and Geriatric Depression Scale (GDS). The incidences of adverse events related to CHMs will also be included as secondary outcomes. All outcome measures have to be administered at baseline and directly after the intervention period. All relevant peerreviewed articles with a randomized controlled trial (RCT) design will be included. Case reports, reviews, prospective or retrospective cohort studies, conference abstracts, and studies not written in English or Chinese will be excluded. When articles reported an overlap in the sample of participants the article with the largest sample was included.

Condition being studied: Mild cognitive impairment (MCI), as a common neurodegenerative aging disease representing an intermediate stage between normal cognitive functioning and dementia, has imposed a significant burden for both patients and caregivers. However, there are no recommended pharmacological or non-pharmacological treatments for MCI. Maintaining satisfactory and extensive symptomatic control in $\mathrm{MCl}$ has been a huge challenge in the management of $\mathrm{MCl}$. Chinese herbal medicines (CHMs), applied widely to treat $\mathrm{MCl}$ in hospitals in China for decades, have gained significant research attention from the global medical community due to their potential as novel treatments for $\mathrm{MCl}$. Chinese Medicine (TCM) has led to a theory for an independent $\mathrm{MCl}$ system. The pathogenesis of such impairment involves deficiency, phlegm, and stagnation and involves a range of organs, including the brain, kidneys, heart, liver, and spleen, leading to the realization that CHMs can improve $\mathrm{MCl}$. Clinical research has shown that CHMs can improve the neuropsychological scale score of patients, and the patient's quality of life. Research has also suggested that CHMs can inhibit the generation of $A \beta$, prevent amyloid pathology, regulate the metabolism of cholinergic neurotransmitters, and so on. Thus, we conducted this study to further evaluate the efficacy and acceptability of CHMs in the treatment of $\mathrm{MCl}$ through meta-analysis and trial sequential analysis (TSA).

\section{METHODS}

Participant or population: Participants diagnosed with $\mathrm{MCl}$ by any proper clinical criteria not caused by traumatic brain injury or space-occupying lesion will be included. There is no restriction on age, sex, race, or region of the enrolled participants.

Intervention: Interventions in the treatment group will include any kinds of orally administered CHMs such as prescription and Chinese patent medicines in the form of liquids, pills, powders, granules, or capsules as mono-therapy. The control group was defined as patients who were assigned to receive placebo. CHMs in combination with other conservative treatments such as cognitive rehabilitation and oral drugs, and other Traditional Chinese medicine (TCM) treatments such as intravenous medication, acupuncture, and massage for cognitive deficit will be excluded. The concomitant use of 
medication for noncognitive disorders will be allowed.

Comparator: The control group was defined as patients who were assigned to receive placebo.

Study designs to be included: All relevant peer-reviewed articles with a randomized controlled trial (RCT) design will be included. Case reports, reviews, prospective or retrospective cohort studies, conference abstracts, and studies not written in English or Chinese will be excluded. When articles reported an overlap in the sample of participants the article with the largest sample was included.

Eligibility criteria: Participants diagnosed with $\mathrm{MCl}$ by any proper clinical criteria not caused by traumatic brain injury or spaceoccupying lesion will be included. There is no restriction on age, sex, race, or region of the enrolled participants.

Information sources: We will apply extensive strategies for preliminary literature search in PubMed, Embase, Cochrane Library, China National Knowledge Infrastructure (CNKI), China Biological Medicine (CBM), Chinese Science and Technology Periodical Database (VIP), and Chinese Medicine Premier (Wanfang) databases to identify relevant articles with no limits on publication date. Through both combined Medical subject headings (MeSH) and textterms followed by Boolean logical operators, an exhaustively search was executed by using the following MeSH terms with the limitation of Chinese and English Ianguage: "Cognitive Dysfunctions", "Mild cognitive impairment", "Cognitive impairment", "Mild Neurocognitive Disorder", "Cognitive Decline", "Randomized controlled trials", "Drugs, Chinese Herbal", as well as additional relevant conceptual keywords. References of studies with potential relevance will be manually checked in case of missing a few underlying eligible articles. We will also search http:// www.clinicaltrials.gov and screened the bibliographies to identify any potentially eligible studies.

Main outcome(s): The change in global cognitive function scores will be applied as the primary outcome measure, derived from MMSE, MoCA, and ADAS-cog scales. Secondary outcomes include assessments of ADL and mood which might be measured by the Bayer ADL, Erlangen ADL, Alzheimer Disease Cooperative Study (ADCS) ADL, the Disability Assessment for dementia (DAD) ADL scales, and Geriatric Depression Scale (GDS). The incidences of adverse events related to CHMs will also be included as secondary outcomes. All outcome measures have to be administered at baseline and directly after the intervention period.

Quality assessment / Risk of bias analysis: Three researchers (S.C.G., L.M.Z. and C.X.Y.) will independently assess risk of bias with use of the Cochrane Collaboration's tool. Risk of bias at trial level is reported in 6 domains: 1) selection bias (random sequence generation and allocation concealment); 2) performance bias (blinding of participants and personnel); 3) detection bias (blinding of outcome assessment); 4) attrition bias (incomplete outcome data); 5) reporting bias (selective reporting); and 6) other bias including financial and academic bias. Each domain will be rated as low, high, or unclear, and a total risk of bias judgment is based on the assessment of all domains. Trials with low risk of bias for all key domains are judged as low risk of bias. Trials with low risk of bias in all domains except blinding of participants and personnel are adjudicated as overall intermediate risk of bias. Trials with high risk of bias for greater than or equal to one key domain are judged as at high risk of bias. We plan to base our main conclusions on the results from trials with overall low or intermediate risk of bias in case no trials were adjudicated as having overall low risk of bias. Differences will be discussed with a third researcher (R.R.Z.) until consensus is reached. 
Strategy of data synthesis: We will report continuous outcomes as mean differences (MDs) with standard deviations (SDs) and dichotomous outcomes as relative risks (RRs) with 95\% confidence intervals (Cls). The primary outcome of the change in global cognitive function scores will be treated as continuous variables and MDs with $95 \% \mathrm{Cls}$ between treatment and control groups will be estimated. Intention to treat (ITT) analyses will be reported if available. All $p$ values are two-tailed and considered statistically significant if less than 0.05 . Statistical heterogeneity between the trials will be assessed primarily by inspecting Forest plots for heterogeneity, secondarily using the inconsistency (I2) and diversity (D2) statistics with thresholds as suggested by the Cochrane Handbook, with 12 values of $0 \%$ to $25 \%, 25 \%$ to $75 \%$, and $75 \%$ to $100 \%$ representing low, moderate, and substantial heterogeneity. We will use random-effects models (assuming that the true intervention effects in the included trials are not identical but follow normal distribution) or fixed-effects models (assuming that the true effect of the intervention in both direction and magnitude is fixed across included trials) across outcomes according to the heterogeneity and report the most conservative estimates with the widest Cls. Fixed-effects models will be applied if there is no evidence of heterogeneity; otherwise, random-effects models will be applied.

Subgroup analysis: We plan to conduct five subgroup analyses of the included trials to evaluate the influences of the following factors on primary outcome: 1) patient age; 2) type of $\mathrm{CHM}$; 3) MCI duration; 4) sample size; 5) cognitive function scores at the baseline. Heterogeneity in the subgroup analyses is assessed using the Chi2 test with $p=0.05$ considered significant. And only subgroup analyses showing a statistically significant test of interaction ( $p$ $<0.05$ ) will be considered to provide evidence of an intervention effect.

Sensitivity analysis: If necessary, a leaveone-out sensitivity analysis will be performed to evaluate the main trials demonstrating a substantial impact on the inter-study heterogeneity. If there is no qualitative change in the combined effect, the results are stable.

\section{Country(ies) involved: China.}

Keywords: mild cognitive impairment, Chinese herbal medicines, meta-analysis, trial sequential analysis, protocol.

\section{Contributions of each author:}

Author 1 - Si-Chun Gu.

Author 2 - Li-Min Zhang.

Author 3 - Chun-Xu Wang.

Author 4 - Yan-Jie Qu.

Author 5 - Jing-Yi Ma.

Author 6 - Rong-Rong Zhen.

Author 7 - Chao Gu.

Author 8 - Can-Xing Yuan. 